Moriña, A., Márquez, C. \& Álvarez, E. (2020). Redes Personales de Estudiantes con Discapacidad en la Universidad. Revista Electrónica Interuniversitaria de Formación del Profesorado, 23(2), 209-224.

DOI: https://doi.org/10.6018/reifop.410261

\title{
Redes Personales de Estudiantes con Discapacidad en la Universidad
}

Anabel Moriña1, Carmen Márquez², Encarnación Álvarez ${ }^{1}$

${ }^{1}$ Universidad de Sevilla; ${ }^{2}$ Universidad Autónoma Madrid

\section{Resumen}

En este artículo se analizan las redes personales de estudiantes universitarios españoles con discapacidad. El propósito fue realizar un análisis de sus redes personales para conocer cómo éstas contribuían al acceso y permanencia en la universidad. Para construir las redes de los estudiantes se realizaron entrevistas semiestructuradas en profundidad y nos apoyamos en Ucinet y Netdraw para su representación. Los resultados fueron organizados en dos apartados: tamaño y composición de las redes personales e identificación de personas o estructuras que facilitan u obstaculizan las trayectorias universitarias. En las conclusiones se plantea que, para estos estudiantes, contar con redes informales fue fundamental, siendo la familia el principal apoyo.

\section{Palabras clave}

Enseñanza Superior; Discapacidad; Redes personales; Análisis de redes personales.

\section{Personal Networks of Students with Disabilities at University}

\section{Abstract}

This article analyzes the personal networks of university students with disabilities. The purpose was to analyze their personal networks to know how they contributed to the access and permanence at university of these students. In order to build student networks,

\section{Contacto:}

Carmen Márquez. Universidad Autónoma de Madrid.carmen.marquez@uam.es

Artículo vinculado a los siguientes proyectos de investigación: Análisis de las Aulas Universitarias Andaluzas desde la Perspectiva de Estudiantes con Discapacidad (P11-SEJ-7255) (Ministerio de Economía y Competitividad de España, proyecto I+D); Barreras y Ayudas que los Estudiantes con Discapacidad Identifican en la Universidad (EDU2010-16264) (Junta de Andalucía, Proyecto de Excelencia I+D). 
we carried out in-depth semi-structured and we relied on Ucinet and Netdraw for their representation. The results were organized in two parts: size and composition of the personal networks of the students; and people who facilitate or obstruct the university trajectories. In the conclusions we suggested that, since other university structures did not respond to the needs of these students, considering the family as the main support.

\section{Key words}

Higher education; disability; personal networks; analysis of social networks.

\section{Introducción}

El perfil del alumnado universitario es cada vez más diverso, estando presentes estudiantes que, tradicionalmente, habían estado poco representados (Hitch, Susie y Nihill, 2015; Thomas, 2016), como es el caso de personas con discapacidad (Seale, 2017). Esta diversidad, junto a otras transformaciones como los enfoques de enseñanza centrados en el alumnado y la incorporación de nuevas tecnologías han demandado que la universidad deba plantearse y poner en marcha políticas y acciones que respondan a las nuevas necesidades que surgen en este contexto de cambio.

En los últimos años, se está demandando que universidad sea inclusiva (Gairín, 2014). Tanto declaraciones internacionales (Estrategia Europea 2020 de Unión Europea, 2010; Agenda 2030 de Naciones Unidas, 2015) como normativas nacionales (Ley Orgánica de Universidades Españolas 4/2007 o Ley General de Derechos de las Personas con Discapacidad y su Inclusión Social, 1/2013) reconocen la necesidad de avanzar hacia la inclusión universitaria. En este contexto se entiende que la educación inclusiva es un derecho básico y una cuestión de justicia social. Los sistemas universitarios deben revisar sus prácticas para garantizar el aprendizaje y participación de todo el alumnado (Valee, 2017).

Este discurso pro inclusión no ha estado exento de propuestas que han contribuido a que la universidad camine en esta dirección. Entre ellas destacan la creación de oficinas de apoyo a la discapacidad o los planes de orientación y acción tutorial. Sin embargo, no resulta suficiente con garantizar el acceso y las acciones encaminadas a este fin, se precisa prestar atención a la progresión y éxito universitario (Gibson, 2015; Thomas, 2016; Wilson et al., 2016).

Diferentes estudios han identificado barreras que dificultan la permanencia universitaria de los estudiantes con discapacidad (Clouder, Adefila, Jackson, Opie y Odedra, 2016; Hopkins, 2011; O’ Byrne, Jagoe y Lawler, 2019; Odame, Opoku, Nketsia y Nanor, 2019). Entre los obstáculos que encuentran las personas con discapacidad, cabe mencionar las barreras arquitectónicas en espacios y edificios universitarios, la burocratización de trámites administrativos o las metodologías de aula que no facilitan la inclusión. Precisamente, las prácticas en el aula son identificadas como la principal dificultad de permanencia. La actitud del profesorado también ha sido reconocida como barrera para el aprendizaje y participación, destacándose la actitud negativa, la falta de sensibilización, la necesidad de formación en cuestiones metodológicas, el desuso o inadecuado uso de las TIC, el desconocimiento sobre la discapacidad o las necesidades derivadas de ésta (Macías, Aguilera, Rodríguez y Gil, 2019; Osborne, 2019; Strnadová, Hájková y Květoňová, 2015).

Paralelamente, se ha identificado personas o estructuras que actúan como facilitadores de las trayectorias educativas del alumnado con discapacidad. Es el caso del 
apoyo familiar (Riddell, Tinklin y Wilson, 2005; Skinner, 2004), de las amistades o iguales (Gibson, 2012; Riddell et al., 2005), del profesorado a través de su conocimiento y amistad (Rodríguez-Martín y Álvarez-Arregui, 2014; Yusof, Chan, Hillaluddin, Ahmad y Saad, 2019) o del apoyo de los servicios de atención al alumnado con discapacidad (Riddell et al., 2005). Incluso, desde el discurso de la resiliencia se han identificado como apoyo las propias estrategias personales que los estudiantes implementan para hacer frente a sus dificultades (Prowse, 2009).

Desde los planteamientos de la teoría de red, hace más de tres décadas, Whittaker y Garbarino (1983) hicieron referencia a la red de apoyo social como un conjunto de relaciones interconectadas entre personas o grupos que proporcionan refuerzo para hacer frente a la vida diaria. En ocasiones, estas redes se convierten en sistemas de apoyo social y constituyen un elemento indispensable para la salud y bienestar del individuo (Paredes Fernández-Cid y Ruiz, 2012). Igualmente, se les atribuye una contribución activa en la conformación de la resiliencia y en el aumento de la capacidad de adaptación ante el cambio ambiental (Tompkins y Adger, 2004).

Las redes personales pueden ser redes informales y surgir como resultado de las interacciones entre miembros de una familia, amigos o compañeros de estudio o trabajo. Este tipo de redes forman parte de las redes naturales de apoyo (Duggan y Linehan, 2013; Echeita, Simón, Sandoval y Monarca, 2013). También pueden ser redes formales o vinculadas a las relaciones generadas en contextos de ayuda profesional. En el caso de las personas con discapacidad, estas redes están constituidas por miembros de organizaciones de apoyo (como ONCE), de los servicios específicos de las instituciones educativas (servicios de atención a estudiantes con discapacidad) o de las empresas en las que trabajan.

De las redes de apoyo se destaca su contribución en los procesos de participación de las personas con discapacidad al convertirse en proveedoras de apoyo y en amortiguadoras del estrés que conllevan ciertos acontecimientos vitales para ellas (Vivaldi y Barra, 2012). Se reconocen como una de las variables más influyentes en los procesos de adaptación y superación de los sucesos complejos experimentados (Suriá, 2017). Estas cualidades han sido reconocidas especialmente en estudios sobre los apoyos laborales de los jóvenes con discapacidad (Suriá, 2015) y estudios centrados en analizar las relaciones de las fuentes apoyo en los entornos laborales (Pérez y Alcover, 2011).

El análisis de redes sociales (ARS), basándose en la teoría de grafos, analiza los patrones de relación entre actores que interactúan entre sí (Sie et al., 2012; Wasserman y Faust, 1994). Esto permite la identificación de las estructuras micro, meso y macro en las que están insertos los actores, incluso cuando las dinámicas no son evidentes ni para ellos ni para los observadores (Maya-Jariego, 2016).

Los datos relacionales pueden medirse a nivel egocéntrico, es decir, centrado en las relaciones que mantiene un individuo con su entorno o a nivel sociocéntrico de manera que nos permita obtener una imagen de los patrones de relaciones en un grupo social definido (Carolan, 2014). Ambas aproximaciones posibilitan conocer las características de las redes y su influencia en el comportamiento social. Complementariamente, los datos relacionales nos aportan información sobre el capital social disponible de cada uno de sus miembros y sobre la diversidad de espacios sociales en los que interactúan (Molina, Lerner y Gómez, 2008).

Entre los campos de estudio del ARS se ha demostrado un interés constante por analizar las relaciones que se establecen en los contextos educativos y su influencia en diferentes procesos educativos. Se han descrito y analizado las relaciones que ofrecen 
apoyo académico en el interior de las aulas (Palacios y Villalobos, 2016), las relaciones de amistad y apoyo social entre estudiantes universitarios (Araya y Maya-Jariego, 2005; Macías, Aguilera, Rodríguez y Gil, 2019) y la influencia de las propiedades estructurales de las redes en el rendimiento educativo (Yao, 2010). Todos estos trabajos han mostraron el significado multidimensional de las relaciones en contextos educativos, donde los estudiantes encontraron afecto, reconocimiento o información e incluso consensuaron reglas de comportamiento y forjaron culturas (Avendano y Díaz, 2014; Santos, 2010).

Este tipo de análisis puede aportar una nueva mirada a los estudios ya realizados sobre barreras y ayudas que encuentra el alumnado con discapacidad en la universidad (Hopkins, 2011), destacándose la aportación de esta investigación que pretende describir las redes de apoyo de los estudiantes con discapacidad durante su trayectoria universitaria.

Desde el ARS nos aproximamos al análisis y visualización de la estructura y composición de las redes personales de cuatro estudiantes universitarios con discapacidad. Los objetivos de investigación que guiaron el trabajo fueron tres:

1) Describir el tamaño y composición de las redes personales de los estudiantes con discapacidad.

2) Analizar qué actores ofrecen apoyo a los estudiantes con discapacidad.

3) Analizar qué actores no ofrecen apoyo a los estudiantes con discapacidad.

\section{Metodología}

\section{Participantes}

El acceso a los participantes fue facilitado por las oficinas de apoyo a la discapacidad de dos universidades públicas y estuvo constituida por cuatro estudiantes universitarios con discapacidad. Con una edad media de 27 años, tres de los participantes eran género femenino y uno masculino. Dos de ellos cursaban un Grado en Educación, uno Grado en Medicina y otro Grado en Ciencias de la Comunicación. Respecto al tipo de discapacidad, la mitad de los participantes $(n=2 ; 50 \%)$ presentaban una discapacidad auditiva y la otra mitad $(n=2 ; 50 \%)$ una discapacidad visual. Para preservar los datos personales de los participantes se han modificado sus nombres en la visualización de las redes personales.

\section{Instrumentos de recogida de información}

Para construir las redes de los estudiantes se realizaron entrevistas semiestructuradas en profundidad sobre las trayectorias universitarias de los participantes. Esta técnica nos ofrecía la posibilidad de analizar sus trayectorias educativas obteniendo información sobre el significado de las relaciones en estos procesos (Emirbayer et al., 1994). En el guión de entrevista incorporamos algunas cuestiones clave para conocer el tipo de apoyo recibido por los estudiantes de cada uno de estos miembros que componían su red personal (Dado et al., 2017). Por ejemplo, algunas de las preguntas que se realizaron fueron: ¿Qué personas te han ayudado en tus estudios universitarios?; ¿cómo influyen los profesores en tu trayectoria universitaria?; Influencia de algún compañero/a en la Universidad, ¿en qué influye?, ¿cómo le ayudan? ¿cómo no le ayudan? ¿Cómo ayuda él/ella a los demás?

\section{Análisis de datos}

La narración de los cuatro estudiantes nos permitió construir las matrices relacionales de cada uno de ellos, extrayendo los verbatims más significativos de sus 
discursos. Los miembros de las redes de apoyo fueron distinguidos en función de dos atributos: relación con el estudiante entrevistado y disponibilidad de apoyo para la consecución del logro escolar. Las relaciones consideradas en el análisis fueron: familiares, compañeros de clase, profesorado, personal de los servicios universitarios de atención a los estudiantes con discapacidad, técnicos o responsables de otros servicios administrativos universitarios y personal externo de apoyo. Esta clasificación de las relaciones nos permitió diferenciar, posteriormente, entre redes formales e informales de apoyo. En cuanto a la disponibilidad de apoyo consideramos: actores que apoyaron al estudiante para la consecución del logro académico y actores que no prestaron apoyo al estudiante. En función de estos datos, distinguimos entre redes de apoyo académico y redes de no apoyo académico.

Los datos se analizaron con Ucinet 6.0 para la obtención los índices de centralidad y la densidad total de las redes. Los datos reticulares fueron combinados con análisis de contenido cualitativo para comprender de manera más profunda la naturaleza de las relaciones de los estudiantes. Con Netdraw se representaros las redes personales, los actores a través de nodos y los vínculos a través de líneas que conectaban a los nodos entre sí. Esta representación gráfica nos proporcionó una visualización intuitiva de los patrones de relaciones grupales.

\section{Resultados}

Los resultados muestran el tamaño y composición de las redes personales de los estudiantes durante sus trayectorias académicas y el apoyo que reciben de estas estructuras.

Describir el tamaño y composición de las redes personales de los estudiantes con discapacidad

Las redes personales que acompañan a los estudiantes con discapacidad durante su periodo universitario mostraron un tamaño bastante reducido. Todas oscilaron entre $n=11$, para tres estudiantes y $n=10$ para uno de los estudiantes (Figura 1). Estas redes estaban compuestas por familiares, compañeros, profesorado, personal de servicios universitarios y profesionales de apoyo externo en diferente proporción. En la red 1, se observó idéntica proporción de familiares, compañeros y profesorado (28\% respectivamente) y una menor presencia del personal de servicios universitario y de apoyo externo ( $8 \%$ respectivamente). En la red 2 se apreció mayor presencia de profesorado (36\%), familiares (28\%), compañeros $(18 \%)$ y de personal de servicios universitarios y de apoyo externo ( $9 \%$ respectivamente). En la red 3, los miembros con mayor presencia fueron los profesionales de los servicios universitarios (45\%) y el profesorado (37\%), seguidos de familiares y compañeros ( $9 \%$ respectivamente). En la red 4 se registró mayor presencia de familiares (30\%), seguidos de compañeros, profesorado y profesionales de los servicios universitarios (20\% respectivamente) y menor presencia de los profesionales de apoyo externos (10\%). En definitiva, en todas las redes se apreció la presencia constante de la familia, convertida en un elemento clave y una alta incidencia de relaciones vinculadas directamente con el ámbito universitario. Esto podía tener su explicación en que los participantes cursaron sus estudios en una modalidad presencial y gran parte de su tiempo se desarrolló en el entorno universitario. 


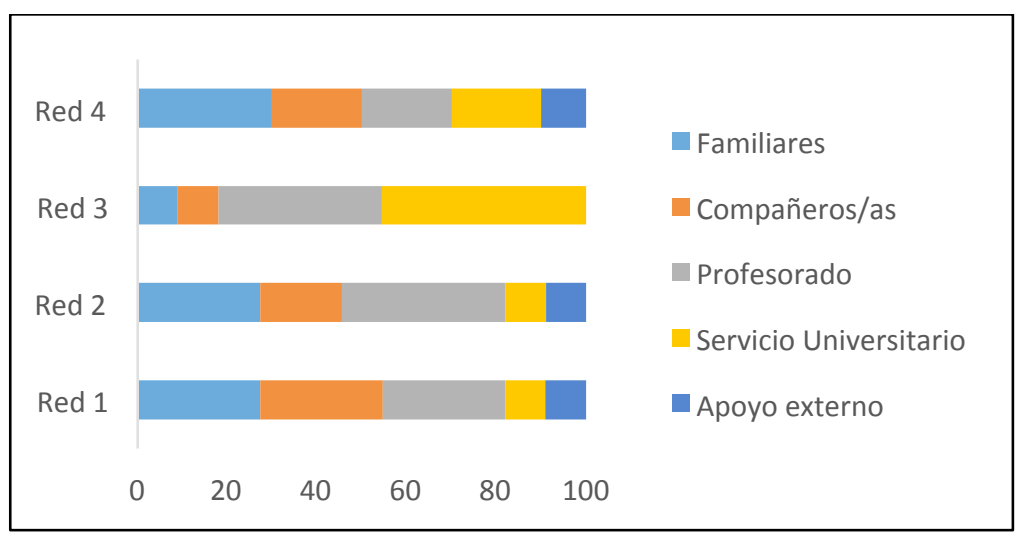

Figura 1. Composición de las redes personales en función de la procedencia del vínculo.

El análisis de la composición de las redes nos permitió distinguir entre redes de apoyo formal e informal. En tres de las redes se destaca una mayor incidencia de las redes informales (Figura 2).

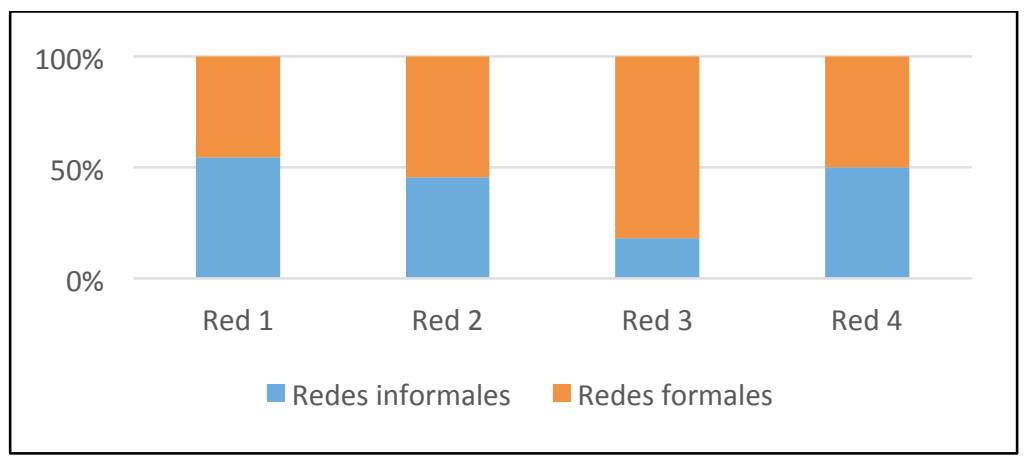

Figura 2. Proporción de redes formales e informales de las redes personales

\section{Analizar qué actores ofrecen apoyo a los estudiantes con discapacidad}

Otro objetivo estaba referido a los miembros con los que pudieron contar los estudiantes con discapacidad durante su periodo de formación universitaria. Para obtener datos en este sentido, diferenciamos en las matrices relacionales entre aquellos miembros que habían prestado apoyo al estudiante y aquellos otros que, a pesar de la solicitud expresa por parte del estudiante, les habían denegado cualquier tipo de apoyo o atención específica. Esta diferenciación propició la identificación de redes de apoyo académico y redes que no ofrecieron este apoyo. Para distinguir esta característica en cada estructuras (de apoyo y de no apoyo) se ha visualizado con diferentes figuras geométricas en el dibujo de red.

\section{Redes de apoyo académico}

Las redes de apoyo académico mostraron una baja densidad, con valores que oscilaron entre el $15 \%$ y $25 \%$. Estas redes fueron, fundamentalmente, proveedoras de apoyo afectivo e instrumental para el estudiante con discapacidad durante su trayectoria universitaria y en todas ellas se identificó la presencia constante de familiares. Fueron precisamente, los miembros de la familia y/o cónyuges quienes ocuparon las posiciones de mayor centralidad en las redes personales de los estudiantes. La familia ofreció a los estudiantes apoyo emocional durante todo el proceso formativo (Figura 3), aunque en 
algún caso, también apoyo instrumental para la realización de algunas actividades de evaluación que el alumnado no podía realizar de manera autónoma (Figura 4).

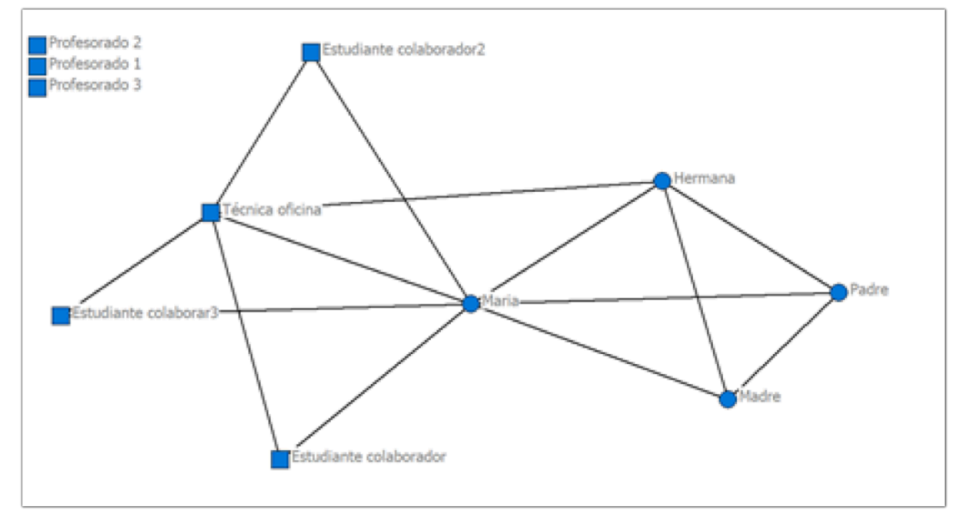

Figura 3. Red de apoyo académico Participante 1

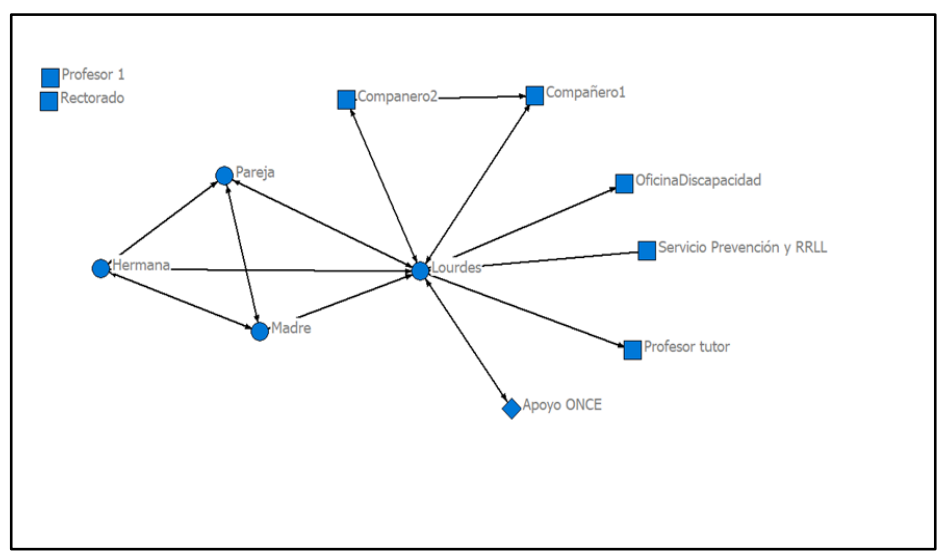

Figura 4. Red de apoyo académico Participante 4

En los siguientes verbatim se ve reflejada la importancia de la incidencia del apoyo que la familia ofreció durante sus trayectorias académicas a estos estudiantes:

"Hasta aquí no he llegado sola, mis compañeros de viaje han sido mi familia. Ellos constituyen el apoyo más incondicional de todos los que ha habido a lo largo mi vida personal y estudiantil" (Participante 1).

"Para mí, uno de mis principales apoyos es quien es ahora mi marido, Antonio. Porque para que yo pudiera entregar las prácticas de periodismo, pues claro, yo las grababa, pero luego, el que me ayudaba a sacar el audio de la grabadora, me lo editaba en Mp3 y se esforzaba porque todos sus conocimientos me ayudaran en mis tareas, era Antonio" (Participante 4).

También fue destacable la presencia de los y las profesionales de los servicios universitarios de atención al alumnado con discapacidad en las redes de apoyo (Figura 5). Estos profesionales se convirtieron en intermediarios del alumnado y el resto de la comunidad educativa en la resolución de conflictos con otros servicios universitarios, comunicación de información específica relativa a su discapacidad al profesorado y la gestión de los convenios de colaboración con los estudiantes colaboradores. 
"La directora del servicio a la discapacidad en aquel entonces fue determinante, ya que fue ella la que convocó al profesorado para ayudarme y estuvo presente" (Participante 3).

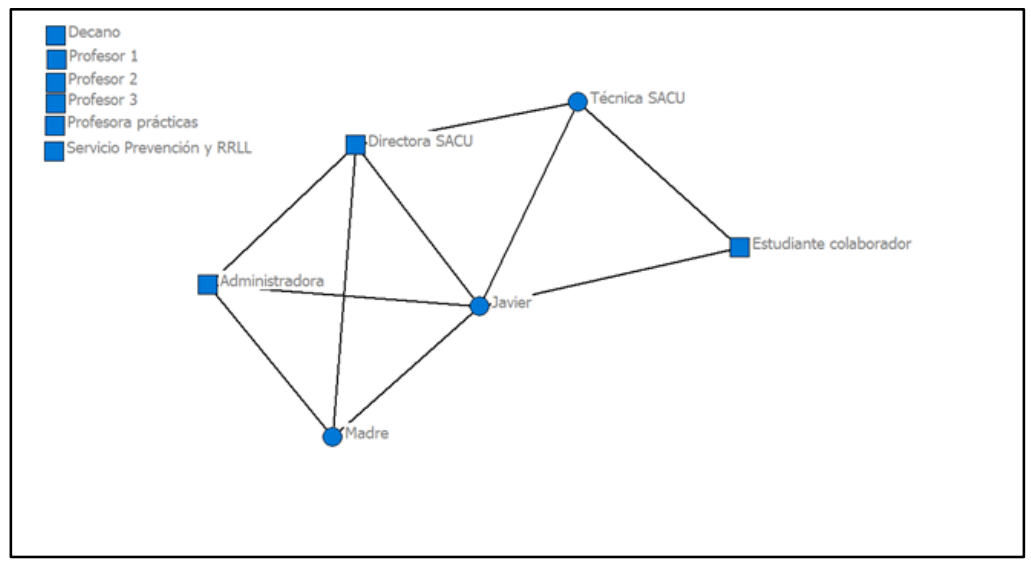

Figura 5. Red de apoyo académico Participante 3

En dos de las redes analizadas se visualizaron también a otros profesionales que realizaron funciones de apoyo instrumental pero pertenecientes a distintas organizaciones o asociaciones de atención a personas con discapacidad externas a la universidad. Es el caso, de los intérpretes de lengua de signos española (ILSE) que, generalmente, pertenecían a asociaciones de personas con discapacidad auditiva o entidades privada y de los profesionales de apoyo y accesibilidad de la Organización Nacional de Ciegos Españoles (ONCE) (Figura 6). Dos de los estudiantes participantes hicieron referencia explícita el apoyo que encontraban en estos profesionales para acceder al currículo o participar en clase.

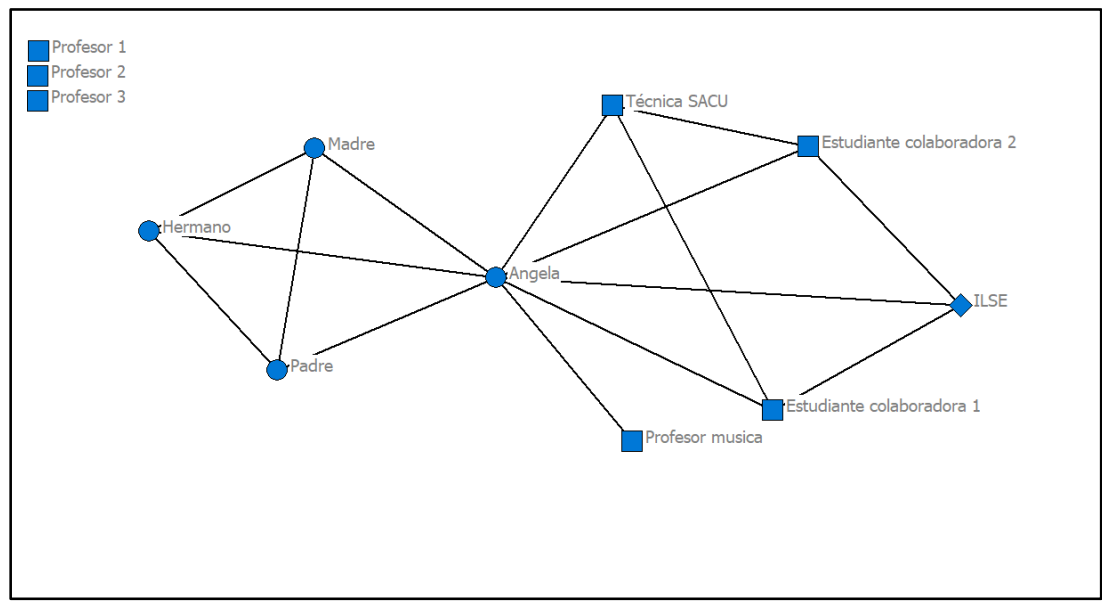

Figura 6. Red de apoyo académico Participante 2

"Otro de los elementos fundamentales que necesitan las personas con diversidad funcional auditiva y que la universidad debería proporcionar durante todo el tiempo es el ILSE, porque para personas como yo es una ayuda fundamental: son nuestros oídos y muchas veces nuestra habla" (Participante 2). 
En cambio, se constató la presencia anecdótica en las redes de apoyo del profesorado y de los compañeros, se circunscribió al apoyo que encontraron en algún profesor particularmente o el apoyo de los estudiantes colaboradores, a quienes les unía una relación formal y gestionada por los servicios de apoyo.

"En mi caso ha sido de mucha ayuda el alumno colaborador que es otro de los recursos que ofrece el SACU a las personas con diversidad funcional auditiva" (Participante 2)

"Por diversas circunstancias he tenido que cambiar cada año de alumno colaborador, con lo cual, han sido tres con las que he contado a lo largo de mi paso por la universidad" (Participante 1)

En definitiva, los resultados analizados nos han permitido visualizar la composición de las redes de apoyo en la etapa universitaria de los estudiantes con discapacidad. En estas estructuras de apoyo destaca la presencia de miembros pertenecientes de familiares y cónyuge (red de apoyo informal) y de profesionales de los servicios de atención a la discapacidad (red de apoyo formal), ya sean propios del ámbito universitario o externo a ellos (Figura 7).

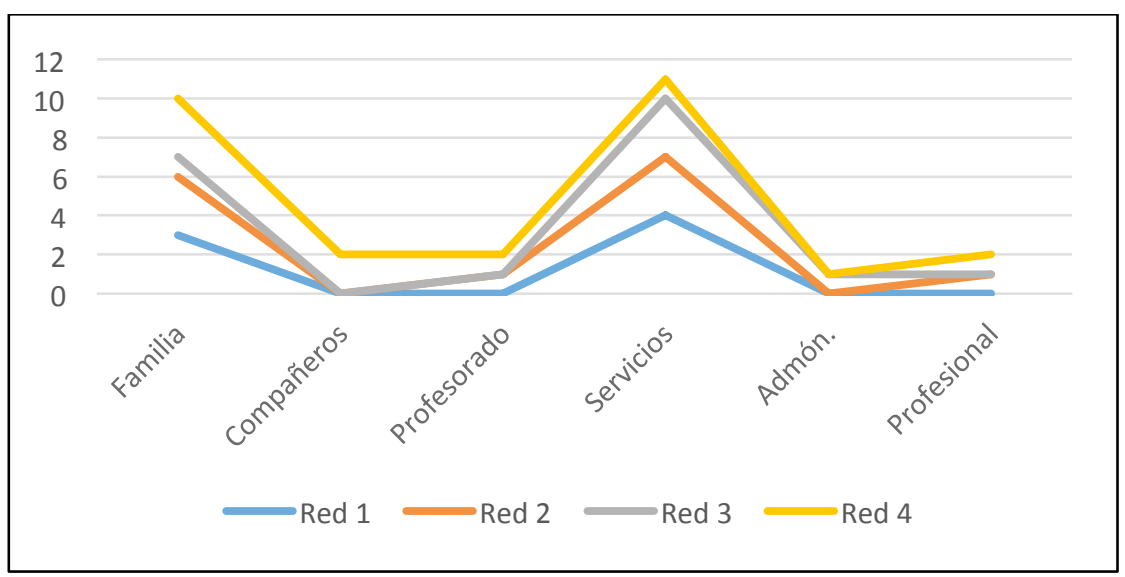

Figura 7. Composición de las redes de apoyo académico

\section{Analizar qué actores no ofrecen apoyo a los estudiantes con discapacidad}

En estas redes se agruparon a los miembros de la red personal que no habían ofrecido apoyo al estudiante con discapacidad, aun cuando había existido la solicitud expresa de ayuda o apoyo por parte del estudiante.

La densidad de estas redes era de menor densidad que las redes de apoyo y sus valores oscilaban entre el $3 \%$ y $5 \%$. Aunque no caben generalizaciones, en el análisis de este tipo de red destacaba la presencia absoluta del profesorado (Figura 8). Los estudiantes reconocieron las dificultades a las que debían hacer frente cuando el profesorado se negaba a ampliar las formas de evaluación, anticipar el material al alumnado o incorporar pautas de accesibilidad al aula.

"Empecé a exponer mi situación a los diferentes docentes. Ahí fue cuando me di cuenta que yo solo no podría hacer frente a esto, pues el profesorado no me tomaba en serio. Incluso alguno me llegó a sugerir que me comprase mejores gafas. $Y$ otra profesora me dijo que no podía pasarme las transparencias por adelantado porque las hacía sobre la marcha" (Participante 3). 
"Recuerdo a un profesor de literatura que me obligaba a hacer los exámenes orales. Yo creía que eso me perjudicaba, pero no me daba otra posibilidad" (Participante 4)

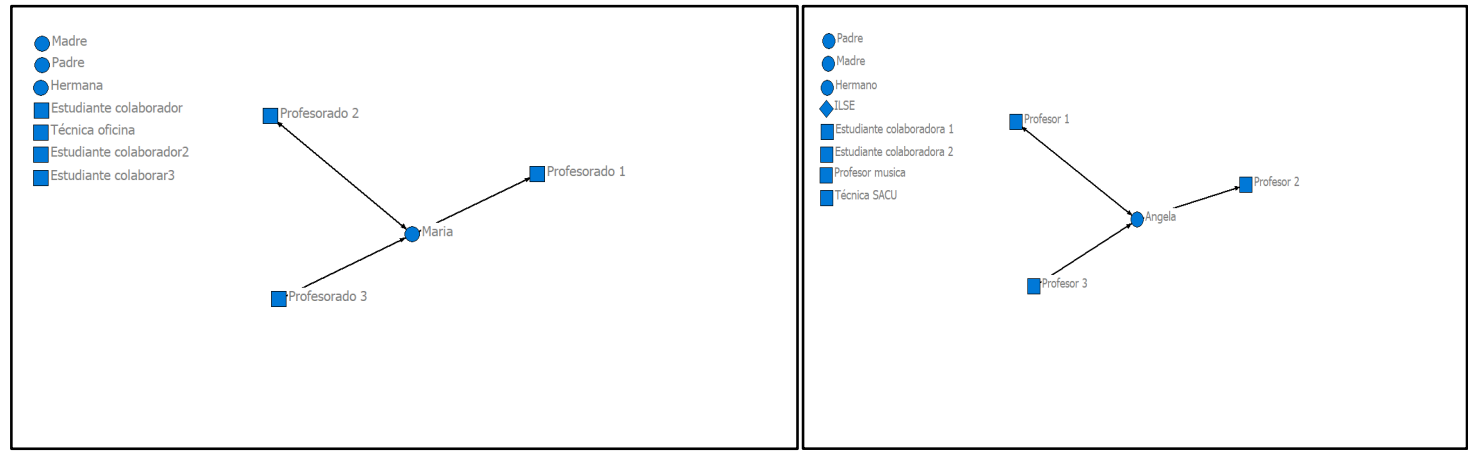

Figura 8. Redes de no apoyo académico

Igualmente, el alumnado reconoció haber tenido dificultades con los servicios universitarios (Rectorado, Decanato o Servicio de Prevención de Riesgos) (Figura 9). Estos servicios no solo no prestaban ningún tipo de apoyo a los estudiantes con discapacidad, sino que, en algunos casos, ni atendían ni respondían a las demandas planteadas por el estudiante.

"Hace unos años me reuní con una persona que trabaja en el servicio de Prevención y Riesgos Laborales, para que se analizara mi Campus porque tengo muchas dificultades con las escaleras que no tienen señales para diferenciar los escalones. Pues me reuní con esta persona, pero aún hoy estoy esperando que se haga esta adaptación". (Javier)

"Me dio problemas un formulario de acceso al doctorado, que me puntuó mal por un problema de accesibilidad y me pareció tan injusto que puse una reclamación a la universidad y ni me constataron". (Lourdes)

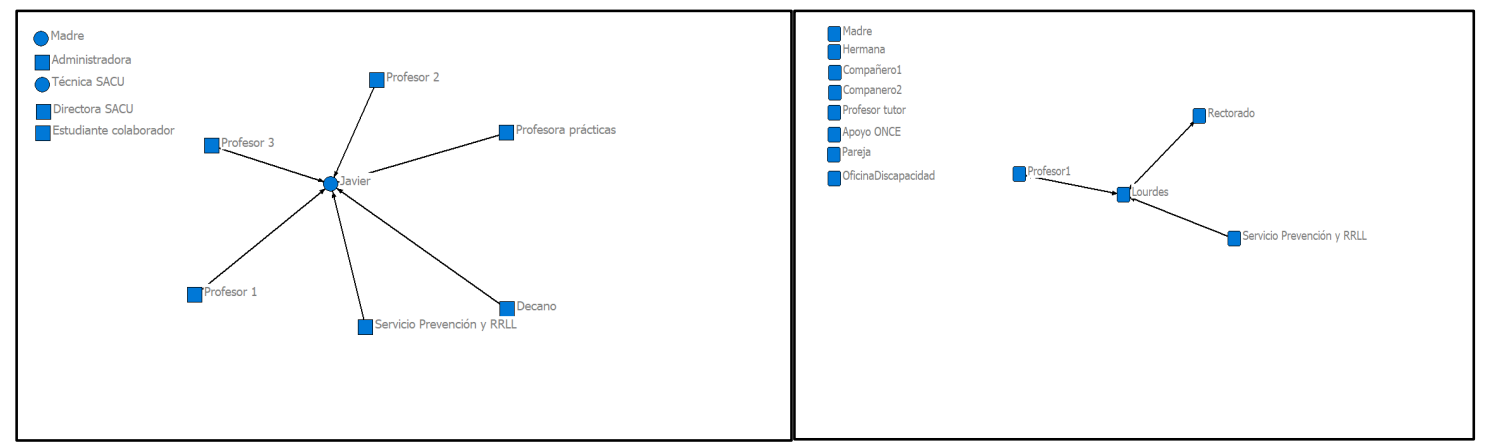

Figura 9. Redes de no apoyo académico

En definitiva, si analizamos quiénes dentro de la estructura universitaria no están ofreciendo apoyo a los estudiantes con discapacidad, podemos apreciar la clara presencia del profesorado y los servicios de la administración universitaria (figura 10). 


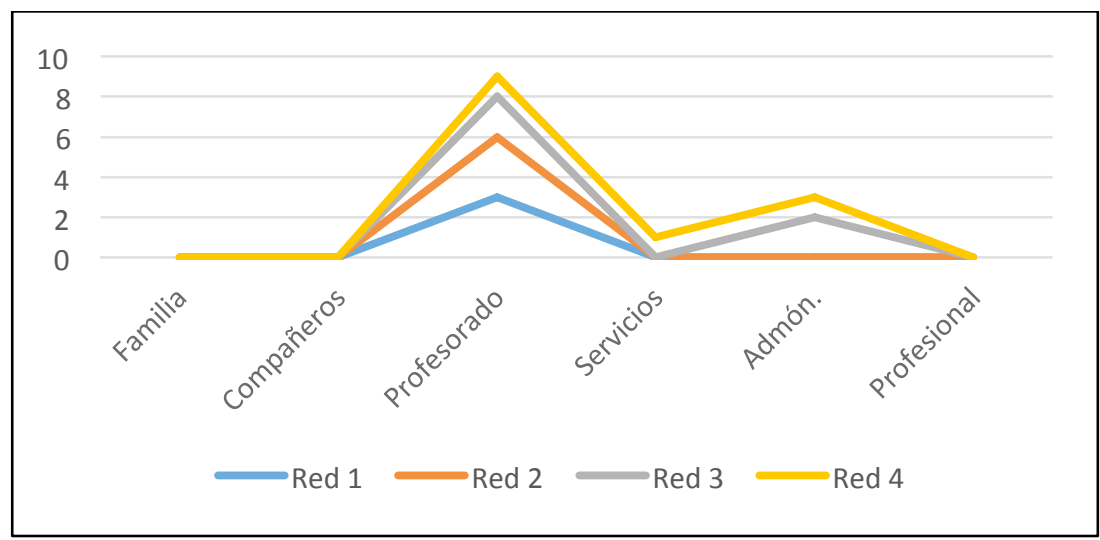

Figura10. Composición de las redes de no apoyo académico

\section{Discusión y conclusiones}

Las redes personales son fundamentales para que las personas con discapacidad puedan acceder, permanecer y finalizar con éxito sus estudios universitarios. Como en otros trabajos se ha estudiado, las trayectorias de estos estudiantes no son fáciles y a menudo están marcadas por una carrera de obstáculos (Gibson, 2012; Hopkins, 2011; Vickerman y Blundell, 2010). En muchos casos, no viven una experiencia universitaria "típica", a causa de las dificultades adicionales, retos y estrategias que tienen que afrontar. Por lo tanto, como concluye este estudio, para estos estudiantes, la familia es el principal referente de apoyo, reconociendo la necesidad para estudiar en la universidad de las redes personales. Este no es un hallazgo nuevo, ya que otros trabajos han llegado a la misma conclusión (Avendano y Díaz, 2014).

En esta investigación, al igual que concluyen Riddell et al., (2005) o Skinner (2004) se evidencia el rol que juega el apoyo de la red de apoyo informal (familia y pareja) en la implicación y desarrollo educativo del estudiante. Aunque se confirma, al igual que en los trabajos de Gibson (2012) y Riddell et al. (2005), la presencia en las estructuras de apoyo de los profesionales de los servicios de apoyo a la discapacidad y de iguales, estos últimos se ven notablemente reducidos en comparación con la influencia de la familia (RodríguezFernández, Droguett y Revuelta., 2012).

No obstante, aunque la familia continúe siendo el mayor proveedor de apoyo para los estudiantes con discapacidad, en la mayor parte de los casos, este apoyo resulta insuficiente para cursar estudios universitarios. Debido a sus necesidades, el estudiante debe buscar fuera de la universidad otros recursos externos que le posibiliten aprender y participar en el aula (por ejemplo, técnicos Ilse y técnicos ONCE), los estudiantes expresan su necesidad de abordar algunas de las dificultades más allá de la red de apoyo informal (Weng, 2016).

Un tema preocupante que se aborda en este estudio es el hecho de cómo miembros de las instituciones universitarias en sus diferentes estructuras (profesores en el aula, gestores en el rectorado, decanato, etc.) están obstaculizando el aprendizaje y la participación del alumnado con discapacidad. Justamente es en la institución universitaria donde el estudiante encuentra su mayor escollo para la consecución del logro académico. Existen evidencias que ponen en cuestión la universidad y cómo ésta está contribuyendo, o más bien, dificultando la inclusión del alumnado con discapacidad (Clouder et al., 2016). Esto demuestra que, si bien en el papel, a través de normativas y reglamentos, se ha hecho eco del discurso inclusivo, en la práctica aún queda un largo camino por recorrer. Como 
demuestra el análisis de redes llevado a cabo en este estudio, el tejido social en el que se encuentra inserto un estudiante también puede convertirse en un factor obstaculizador en su proceso de inclusión (Izquierdo, 2011), protegiendo o poniendo en riesgo el aprendizaje y participación del alumnado. En este sentido, la universidad viene actuado reactivamente, siendo el estudiante el que se ha tenido que adaptar y buscar los apoyos en los contextos informales y formarles externos a la universidad. Una reflexión que nos plantea esta situación es que hubiera sucedido con las trayectorias universitarias de estos cuatro estudiantes si no hubieran contado con las redes de apoyo familiares y por extensión, por qué otros estudiantes con discapacidad no acceden o abandonan prematuramente la universidad. Ésta podría ser una línea de investigación futura en la que estudiar los apoyos informales de personas con discapacidad y cómo estos pueden influir en su acceso y permanencia en la universidad, dado que otras estructuras están fallando.

En definitiva, nuestra investigación ha demostrado que las redes de apoyo social son fundamentales en la adaptación y participación en nuevos contextos. Si hacemos referencia al estudiante con discapacidad, no cabe duda que el acceso al entorno universitario también es un nuevo contexto organizativo, educativo y social al que debe enfrentarse (Tincani, 2004; Thomas, 2008), en la mayor parte de los casos, con dificultades añadidas, como el desconocimiento de los servicios de apoyo disponibles y sus derechos legales en la universidad, o la necesidad una mayor auto-dirección y auto-determinación en sus propias vidas universitarias (Getzel y Colleen, 2008; Hong, 2015). Esta situación justifica la importancia de contar con redes de apoyo social formales e informales que contribuyan al logro académico. Pero no se debe obviar la responsabilidad de la universidad, ya que, si en esta se dieran las condiciones necesarias y fuera realmente una institución inclusiva, el apoyo familiar pasaría a un segundo plano y no sería imprescindible para que el alumnado pudiera acceder y finalizar con éxito sus estudios universitarios.

\section{Referencias}

Araya, R. y Maya-Jariego, I. (2005). Los puentes interlocales: las redes personales de los universitarios alcalareños en Sevilla. En J. I. Porras y V. Espinoza (Eds.). Redes. Enfoques y aplicaciones del análisis de redes sociales (pp. 183-213). Santiago de Chile: Universidad Santiago de Chile y Universidad Bolivariana.

Avendano, C. y Díaz, J. (2014). El proceso de integración educativa desde la perspectiva de cinco jóvenes universitarios con discapacidad visual de Concepción. Estudios Pedagógicos, 40(2), 27-44. Recuperado de http://dx.doi.org/10.4067/S071807052014000300003.

Carolan, B. (2014). Social network analysis and education: Theory, methods and applications. Thousand Oaks: SAGE Publications.

Clouder, L., Adefila, A., Jackson, C., Opie, C. y Odedra, S. (2016). The discourse of disability in higher education: insights from a health and social care perspective. International Journal of Educational Research, 79, 10-20. https://doi.org/10.1016/j.ijer.2016.05.015

Dado, M. y Bodemer, D. (2017). A review of methodological applications of social network analysis in computer-supported collaborative learning. Educational Research Review, 22, 159-180. doi: 10.1016/j.edurev.2017.08.005

Duggan, C. y Linehan, C. (2013). The role of 'natural supports' in promoting independent living for people with disabilities; a review of existing literature. British Journal of Learning Disabilities, 41, 199-207. https://doi.org/10.1111/bld.12040 
Echeita, G., Simón, C., Sandoval, M. y Monarca, H. (2013). Cómo fomentar las redes naturales de apoyo en el marco de una escuela inclusiva: propuestas prácticas. Sevilla: Editorial MAD.

Emirbayer, M. y Goodwin, J. (1994). Network Analysis, Culture and the Problem of Agency. The American Journal of Sociology, 99(6), 1411-1454. https://doi.org/10.1086/230450

Gairín, J. (Coord.) (2014). Colectivos vulnerables en la Universidad. Reflexiones y propuestas para la intervención. Madrid: Wolters Kluwer España.

Gallar, M. (2006). Promoción de la salud y apoyo psicológico al paciente. Madrid: Thomson Learning Paraninfo.

Getzel, E. y Colleen, T. (2008). Experiences of college students with disabilities and the importance of self-determination in higher education settings. Career Development for Exceptional Individuals, 31, 77-84. doi: 10.1177/0885728808317658

Gibson, S. (2012). Narrative accounts of university education: socio-cultural perspectives of students with disabilities. Disability y Society, 27(3), 353-369. doi: 10.1080/09687599.2012.654987.

Gibson, S. (2015). When rights are not enough: What is? Moving towards new pedagogy for inclusive education within UK universities. International Journal of Inclusive Education, 19 (8), 875-886. doi: 10.1080/13603116.2015.1015177

Macías, M.E., Aguilera, J.L., Rodríguez, M. y Gil, S. (2019). Un estudio transversal sobre las actitudes de los estudiantes de pregrado y máster en ciencias de la educación hacia las personas con discapacidad. Revista Electrónica Interuniversitaria de Formación del Profesorado, 22(1), 225-240.

Hitch, D., Susie, M. y Nihill, C. (2015). Inclusive pedagogy in Australian universities: A review of current policies and professional development activities. The International Journal of the First Year in Higher Education, 6(1),135-145. doi: 10.5204/intjfyhe.v6i1.254

Hong, B. (2015). Qualitative Analysis of the barriers college students with disabilities experiences in higher education. Journal of College Student Development, 56(3), 209226. doi: $10.1353 /$ csd.2015.0032

Hopkins, L. (2011). The path of least resistance: a voice-relational analysis of disabled students' experiences of discrimination in English universities. International Journal of Inclusive Education, 15, 711-727. doi: 10.1080/13603110903317684

Izquierdo, R. (2011). Jóvenes vulnerables a la entrada del mercado de trabajo: visualización de ego-redes en Talleres Prelaborales de la Zona Norte de Sevilla. Redes: Revista hispana para el análisis de redes sociales, 21(12), 571-603. https://doi.org/10.5565/rev/redes.427

Ley Orgánica 4/2007, de 12 de abril, por la que se modifica la Ley Orgánica 6/2001, de 21 de diciembre, de Universidades (Boletín Oficial del Estado, 39, Viernes 13 abril 2007).

Ley Orgánica 4/2007, de 12 de abril, por la que se modifica la Ley Orgánica 6/2001, de 21 de diciembre, de Universidades (Boletín Oficial del Estado, 39, Viernes 13 abril 2007).

Macías, M.E., Aguilera, J.L., Rodríguez, M. y Gil, S. (2019). Un estudio transversal sobre las actitudes de los estudiantes de pregrado y máster en ciencias de la educación hacia las personas con discapacidad. Revista Electrónica Interuniversitaria de Formación del Profesorado, 22(1), 225-240. https://doi.org/10.6018/reifop.22.1.353031 
Maya-Jariego, I. (2016). 7 usos del análisis de redes en la intervención comunitaria. Redes: Revista hispana para el análisis de redes sociales 27(2), 1-10. https://doi.org/10.5565/rev/redes.628

Molina, J.L., Lerner, J. y Gómez, S. (2008). Patrones de cambio de las redes personales de inmigrantes en Cataluña. Redes. Revista hispana para el análisis de redes sociales, 15(4), 36-60. https://www.raco.cat/index.php/Redes/article/view/121672/168135

Naciones Unidas (2015). Transformar nuestro mundo: la Agenda 2030 para el Desarrollo Sostenible. Resolución aprobada por la Asamblea General el 25 de septiembre de 2015. A/RES/70/1, 21 de octubre. Recuperado de https:/www.un.org/pga/wpcontent/uploads/sites/3/2015/08/120815_outcome-document-of-Summit-for-adoptionof-the-post-2015-development-agenda.pdf

O’Byrne, C., Jagoe, C. y Lawler, M. (2019). Experiences of dislexia and the transition to university: A case study of five students at different stages of study. Higher Education Research \& Development. doi: 10.1080/07294360.2019.1602595

Odame, L., Opoku, M., Nketsia, N. y Nanor, B. (2019): University Experiences of Graduates with Visual Impairments in Ghana, International Journal of Disability, Development and Education. doi: 10.1080/1034912X.2019.1681375

Osborne, T. (2019). Not lazy, not faking: teaching and learning experiences_of university students with disabilities, Disability \& Society, 34(2), 228-252. Doi: 10.1080/09687599.2018.1515724

Palacios, D. y Villalobos, C. (2016). Redes académicas al interior de las escuelas chilenas: Un estudio exploratorio utilizando Modelos Exponenciales de Grafos Aleatorios (ERGM). Revista hispana para el análisis de redes sociales, 27(2), 33-44. https://doi.org/10.5565/rev/redes.631

Paredes, M., Fernández-Cid, M. y Ruiz, J. (2012). Prevención de riesgos laborales entre las personas con discapacidad intelectual en los centros especiales de empleo. Cuadernos de Trabajo Social, 25(1), 249-260. https://doi.org/10.5209/rev_CUTS.2012.v25.n1.38448

Pérez, V. y Alcover, C. (2011). Apoyo social, satisfacción laboral y abandono en trabajadores con discapacidad. Boletín de Psicología, 102, 23-41. Recuperado de https://www.uv.es/seoane/boletin/previos/N102-2.pdf

Prowse, S. (2009). Institutional construction of disabled students. Journal of Higher Education Policy and Management, 3, 89-96. doi: 10.1080/13600800802559302

Real Decreto Legislativo 1/2013, de 29 de noviembre, por el que se aprueba el Texto Refundido de la Ley General de derechos de las personas con discapacidad y de su inclusión social (Boletín Oficial del Estado, 289, martes 3 diciembre 2013).

Riddell, S., Tinklin, T., y Wilson, A. (2005). Disabled students in Higher Education. London: Routledge.

Rodríguez-Fernández, A., Droguett L. y Revuelta, L. (2012). Ajuste escolar y personal en la adolescencia: El papel del autoconcepto académico y del apoyo social percibido. Revista de Psicodidáctica, 17(2), 397-414. http://dx.doi.org/10.1387/RevPsi

Rodríguez-Martín, A. y Álvarez-Arregui, E. (2014). Estudiantes con discapacidad en la Universidad. Un estudio sobre su inclusión. Revista Complutense de Educación, 25(2), 457-479. 
Santos, M. (2010). Análisis de las redes sociales y rendimiento académico: lecciones a partir del caso de Estados Unidos. Debates en Sociología, 35, 7-44. Recuperado de http://revistas.pucp.edu.pe/index.php/debatesensociologia/article/view/2125

Seale, J (2017). From the voice of a 'socratic gadfly': a call for more academic activism in the researching of disability in postsecondary education". European Journal Of Special Needs Education, 3(1), 53-169. doi:10.1080/08856257.2016.1254967.

Sie, L., Ullmann. T., Rajagopal, K., Cela, K., Bitter-Rijpkema, M. y Sloep, P. (2012). Social network analysis for technology-enhanced learning: Review and future directions. International Journal of Technology Enhanced Learning, 4, 172-190. Recuperado de http://oro.open.ac.uk/37829/

Skinner, M. (2004). College Students with Learning Disabilities Speak Out: What It Takes to Be Successful in Postsecondary Education. Journal of Postsecondary Education and Disability, 17(2), 91-104. Recuperado de https://eric.ed.gov/?id=EJ876005

Suriá, R. (2017). Redes virtuales y apoyo social percibido en usuarios con discapacidad: análisis según la tipología, grado y etapa en la que se adquiere la discapacidad. Escritos de Psicología, 10(1), 31-40. http://dx.doi.org/10.5231/psy.writ.2017.21403.

Suriá, R. (2015). Perfiles resilientes y calidad de vida en personas con discapacidad sobrevenida por accidentes de tráfico. Gaceta Sanitaria, 29, 55-59. https://doi.org/10.1016/j.gaceta.2015.01.016

Strnadová, I., Hájková, V. y Květoňová, L. (2015). Voices of university students with disabilities: inclusive education on the tertiary level - a reality or a distant dream? International Journal of Inclusive Education, 19(10), 1080-1095. doi: 10.1080/ 13603116.2015.1037868.

Tincani, M. (2004). Improving Outcomes for College Students with Disabilities: Ten Strategies for Instructors. College Teaching, 52(4), 128-133. doi: 10.3200/ CTCH.52.4.128133

Tompkins, E. y Adger, W. N. (2004). Does adaptive management of natural resources enhance resilience to climatic change? Ecology and Society, 9(2), 1-14. Recuperado de http://www.ecologyandsociety.org/volg/iss2/art10/

Thomas, L. (2008). Learning and teaching strategies to promote students retention and success. En G. Crosling., L. Thomas., y M. Headney (Eds.). Improving student retention in higher education (pp. 69-81). Nueva York: Routledge.

Thomas, L. (2016). Developing inclusive learning to improve the engagement, belonging, retention, and success of students from diverse groups. En M. Shah., A. Bennett., y E. Southgate (Eds.). Widening higher education participation. A global perspective (pp. 135-159). Oxford: Elsevier.

Unión Europea (2010). European Disability Strategy 2010-2020: A Renewed Commitment to a Barrier-Free Europe. Recuperado de http://eurlex.europa.eu/LexUriServ/LexUriServ.do?uri=COM:2010:0636:FIN:en:PDF

Valee, D. (2017). Student engagement and inclusive education: reframing student engagement. Internatinal Jorunal of Inclusive Education, 21(9), 920-937. doi: 10.1080/13603116.2017.1296033

Vickerman, P. y Blundell, M. (2010). Hearing the voices of disabled students in higher education. Disability and Society, 25, 21-32. doi:10.1080/09687590903363290. 
Vivaldi, F. y Barra, E. (2012). Bienestar Psicológico, Apoyo Social Percibido y Percepción de Salud en Adultos Mayores. Terapia Psicológica, 30(2), 23-29. http://dx.doi.org/10.4067/S0718-48082012000200002

Wasserman, S. y Faust, K. (1994). Social network analysis. Cambridge: Cambridge University Press.

Weng, S. (2016). Leadership in an Asian American Community in the South: The Development of an Informal Support Network to Increase Access to Services. Nonprofit and Voluntary Sector Quarterly, 45(1), 5-22. doi: 10.1177/0899764014561797.

Wilson, K. L., Murphy, K. A., Pearson, A. G., Wallace, B. M., Reher, V. G. S. y Buys, N. (2016). Understanding the early transition needs of diverse commencing university students in a health faculty: informing effective intervention practices, Studies in Higher Education, 41(6), 1023-1040, doi: 10.1080/03075079.2014.966070

Whittaker, J. y Garbarino, J. (1983). Social Support Networks. Informal Helping in de Human Services. New York: Aldine.

Yao, Y. (2010). Comparing two discussion designs in terms of student online interactions. In proceedings of the 2nd international conference on education technology and computer (ICETC), China (Vol. 1, pp. V1eV219). doi:10.1109/ICETC.2010.5529264.

Yusof, Y., Chan, C., Hillaluddin, A., Ahmad, F. y Saad, Z. (2019). Improving inclusion of students with disabilities in Malaysian higher education. Disability \& Society. Doi: 10.1080/09687599.2019.1667304 